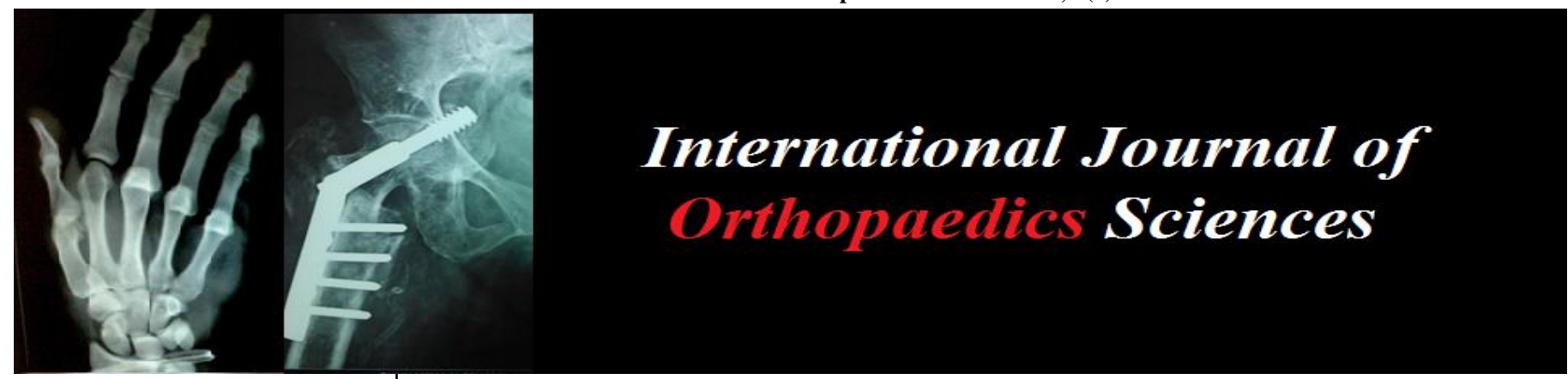

E-ISSN: 2395-1958

P-ISSN: 2706-6630

IJOS 2021; 7(1): 826-830

(C) 2021 IJOS

www.orthopaper.com

Received: 10-11-2020

Accepted: 12-12-2020

Dr. Saumya Sood MBBS MS Orthopaedics,

FMMCH, Mangalore,

Karnataka, India

Dr. Ronald Menezes

HOD MS DNB orthopaedics,

Mangalore, Karnataka, India

Corresponding Author:

Dr. Saumya Sood

MBBS MS Orthopaedics,

FMMCH, Mangalore,

Karnataka, India

\section{Lateral versus posterolateral approach of hemiarthroplasty for fracture neck of femur using bipolar prosthesis}

\section{Dr. Saumya Sood and Dr. Ronald Menezes}

DOI: https://doi.org/10.22271/ortho.2021.v7.i1m.2576

Abstract

Introduction: The purpose of the study is to compare functional outcome, early surgical complications and component alignment between lateral and posterolateral approach of hemiarthroplasty using bipolar prosthesis in fracture neck of femur.

Method: Data was collected from 60 patients, who had fracture neck of femur and aged 55 years and above, between the period April 2018 to October 2019, with minimal sample size of 30 in each group. It is a prospective observational study. Aim of the study is to compare the lateral and posterolateral approaches for hemiarthroplasty.

Results: In a total pool of 60 patients, the mean age of patients in posterolateral group was 70.97 years while that in the lateral group was 73.03 years. Out of the 60 participants, 25 were male patients while 35 female patients. The mean duration of surgery in posterolateral approach in the cemented group (13 patients) was 73.85 minutes, while in uncemented group (17 patients) was 64.65 minutes. In the lateral approach mean duration of surgery in cemented group ( 25 patients) was 73.92 minutes while in uncemented (5 patients) was 65.80 minutes. Average blood loss in posterolateral approach group was $254.67 \mathrm{ml}$ and that in the lateral approach group was $263.00 \mathrm{ml}$. There was no complication of postoperative infection found in either study groups. In our study, leg length discrepancy of $>10 \mathrm{~mm}$ found in $16.7 \%(n=5)$ of posterolateral group and $13.3 \%(n=4)$ in lateral group and was statistically insignificant. Femoral stem positioning was neutral within both posterolateral and lateral surgical approach groups. There was no posterior dislocation in either approach groups. The mean Harris hip score (HHS) at 6 months in posterolateral group was 71.9 with the lowest score of 38.4 and the highest score of 96.2. The mean HHS at 6 months in the lateral group was 66.75 with the lowest score of 47.6 and the highest of 85 . Comparison of the HHS at 6 months between the two groups shows that HHS at 6 months is higher in posterolateral approach group.

Conclusion: There is no significant difference between the lateral and posterolateral approaches in the outcomes of duration of surgery, amount of blood loss, early surgical complications, post-operative prosthesis alignment and the functional outcome. In our study it is concluded that the approach used for HA (hip arthroplasty) depends on the preference and training of the surgeon and results of both the approaches are comparable.

Keywords: HA-hemiarthroplasty, lateral approach, posterolateral approach, harris hip score

\section{Introduction}

Fracture of neck of femur is one of the most common traumatic injuries in elderly, leading to both morbidity and mortality. It is mainly secondary to osteoporosis and falls ${ }^{[1]}$. India is the second most populated country in the world, with $8.6 \%$ of its total population comprising of older adults above the age of 60 years ${ }^{[2]}$. Incidence of fracture of neck of femur is rising continuously due to ageing of the population and urbanization.

HA was first introduced by Moore and Bohlman, with introduction of Austin Moore endoprosthesis. Similar endoprosthesis was introduced by Thompson in 1954. Prosthetic replacement of the femoral head with Austin Moore, Thompson prosthesis and now bipolar prosthesis has undoubtedly played an important role in the treatment of these fractures, especially in those who require immediate mobilization with full weight bearing.

HA is a common treatment for displaced fractures of the femoral neck in the elderly and is associated with better functional outcome (less hip pain, a better hip function) and fewer 
re-operations than internal fixation $[3,4,5]$. HA enables immediate full weight-bearing without the risk of typical complications related to internal fixation, including avascular necrosis and non-union. HA can be done by anterior, lateral, posterolateral and posterior approach.The main surgical approaches are lateral and posterolateral [6, 7]. The posterolateral approach is generally considered to be easy to perform and has less tissue dissection, which leads to shorter operation times and less blood loss. The advantage of a lateral (Hardinge) approach is that it can provide generous exposure of the acetabulum ${ }^{[8,9,10]}$. Each approach has advantages and a different spectrum of complications. Previously conducted studies of hip fracture patients treated with HA indicate that the posterolateral approach increases the risk of hip dislocation and reoperation compared to the lateral approach $[11,12,13]$. The lateral approach, however, may predispose to hematoma formation. Despite the noticeable advancements in surgical interventions, treatment of fracture neck of femur in the elderly remains an area of interest.

\section{Study Methodology}

This study was a prospective observational study, conducted at Father Muller Medical College Hospital, Mangalore, from April 2018 to October 2019. It was conducted on patients above 55 years of age presenting in emergency with fracture neck of femur diagnosed on X-ray, and were planned for HA using bipolar prosthesis. The preference for surgical approach depended upon senior orthopedic surgeon (assistant professor and above). Data was collected for gender, age, surgical approach, type of implant (cemented or uncemented), postoperative infection (upto 2 weeks), duration of surgery, blood loss and post-operative prosthesis component alignment assessed by Leg Length Determination (LLD), femoral stem positioning. The amount of blood loss was calculated as i) suction collected minus total volume of saline used, ii) the total number of mops used in surgery. Mop used was of size $30 * 30$ and on an average $10 \mathrm{ml}$ of blood loss per mop was taken ${ }^{[14]}$. Superficial infections were defined as those wound infections requiring treatment with antibiotics or surgical drainage. Deep infections were those around the prosthesis. LLD was measured by drawing a line transversely connecting inferior borders of acetabular tear drops as the pelvic reference line. Lesser trochanter was used as femoral reference line. The difference between the lines being leg length discrepancy. A measurement of $>10 \mathrm{~mm}$ was defined by convention as unacceptable LLD. The long axis of the stem was compared against the femoral anatomical axis to determine alignment in terms of varus / valgus component. The alignment of a resurfacing arthroplasty should be placed in a relative valgus position of $5^{\circ}-10^{\circ}$. Functional outcome was assessed by HHS. Patients were followed at 2 weeks, 3 months and 6 months interval.

\section{Approach \\ Lateral/ Hardinge's approach}

The patient was positioned either in the supine or lateral position according to the surgeon's choice. A lazy J-incision centering over greater trochanter was made. Fascia lata was divided along skin incision. Tensor fascia lata was retracted anteriorly and gluteus maximus posteriorly. Tendinous junction of gluteus medius and vastuslateralis was exposed. An oblique incision was made over gluteus medius obliquely across the greater trochanter leaving posterior half still attached to the trochanter. This incision was carried proximally over the gluteus medius and distally in line with vastus lateralis. The tendinous insertion of gluteus medius and vastus lateralis was elevated anteriorly. The capsule was visualised and ' $\mathrm{T}$ ' incision was made. The knee was flexed after the limb was externally rotated and adducted. Femoral head was extracted with a cork-screw extractor, taking care not to damage the articular surface of acetabulum. Acetabulum was inspected and all loose debris and bone fragments were removed, along with the remnants of the ligamentum teres. Next attention was turned to preparing the femoral canal. Reaming of the femoral canal was done by hand. The reamer was used in a position of $5-100$ anteversion to ensure that the prosthesis, when inserted will lie with this much degree of anteversion. Then the neck was trimmed to the contour of bipolar prosthesis so as to get a close fit with collar of the prosthesis. Care was taken to maintain correct neck length. Head size of prosthesis was determined by measuring the head of femur, which was removed and measured with a measuring template. A trial reduction with the selected prosthesis was done. Bipolar prosthesis was inserted into the fenestrated canal. Head was manipulated in to acetabulum using standard reduction techniques. Then wound was closed in layers over suction drain.

\section{Posterolateral approach}

The patient was placed in lateral decubitus position. Skin incision was started posterior to the lateral side of greater trochanter and carried distally about $6 \mathrm{~cm}$ along the femoral axis. Proximally incision was slightly curved towards PSIS (posterior superior iliac spine) to a point $6 \mathrm{~cm}$ proximal to greater trochanter. Fascia lata was incised in line with the skin incision. By blunt dissection, the fibres of gluteus maximus were separated, taking care not to disturb superior-gluteal vessels in the proximal part of the exposure. The proximal fibres of gluteus maximus were retracted proximally and the greater trochanter was exposed. A small branch of the sacral plexuses to the quadrates femoris and inferior gamellus, which contains sensory fibers to the joint capsule, was divided. Next, the gamelli and obturator internus (short external rotators) were exposed and divided. The posterior part of the joint capsule was well exposed from distal to proximal along the line of the femoral neck to the rim of the acetabulum. The distal part of the capsule was detached from femur to expose the head of femur. Femoral head was extracted and remaining steps followed in a similar manner as previously described in lateral approach.(short external rotators were closed)

\section{Results}

Out of 60 patients, the mean age of patients in posterolateral group approach was 70.97 years while that in the lateral approach group was 73.03 years. The minimum age found in our study was 55 years and maximum age was 93 years.

Out of the 60 participants, 25 were male patients while 35 female patients. The number of male patients in the posterior approach group was $15(50 \%)$ in the lateral approach group was $10(33.33 \%)$. The number of females in posterolateral group was $15(50 \%)$ and lateral approach group was 20 $(66.66 \%)$. The mean duration of surgery in posterolateral approach in the cemented group (13 patients) was 73.85 minutes, while in uncemented group (17 patients) was 64.65 minutes. In lateral approach, mean duration of surgery in cemented group (25 patients) was 73.92 minutes while in uncemented group (5 patients) was 65.80 minutes. Comparison of duration of surgery between the two groups 
shows that duration of surgery is higher in lateral approach group with a $t$ value of -0.035 and is statistically non significant with a $\mathrm{p}$ value of 0.972. Comparison of the duration of surgery in uncemented HA between the two approach groups shows that duration of surgery is higher in lateral approach group with a $t$ value of -0.601 and is statistically non significant with a $p$ value of 0.55 . The average blood loss in posterolateral approach group was $254.67 \mathrm{ml}$ and that in the lateral approach group was 263.00 ml. Comparison of the blood loss between the two groups shows that blood loss is higher in lateral approach group with a t value of -0.793 and is statistically non- significant with a $p$ value of 0.431 . There was no complication of post-operative infection found in either study groups. In our study, leg length discrepancy of $>10 \mathrm{~mm}$ found in $16.7 \%(\mathrm{n}=5)$ of posterolateral group and in $13.3 \% \quad(n=4)$ in lateral group and was statistically insignificant. The femoral stem positioning was neutral within both posterolateral and lateral surgical approach groups. There was no posterior dislocation in either approach groups. Functional outcome: The mean HHS at 2 weeks in posterolateral approach group was 31.03 with the lowest score being 17.2 and the highest score 42.8 . The mean
HHS in the lateral approach group at 2 weeks was 32.21 with the lowest score of 18 and the highest score of 41.8 . Comparison of the HHS at 2 weeks between the two groups shows that HHS at 2 weeks is higher in lateral approach group with a $t$ value of -0.518 and is statistically non-significant with a $\mathrm{p}$ value of 0.606 The mean HHS at 3 months in posterolateral approach group was 56.25 with the lowest score being 28.4 while the highest being 81.2. The mean HHS at 3 months in the lateral approach group was 52.52 with the lowest being 37.6 while the highest was 67.9. Comparison of the HHS at 3 months between the two groups shows that HHS at 3 months is higher in posterolateral approach group with a $t$ value of 1.199 and is statistically non-significant with a $p$ value of 0.236 . The mean HHS at 6 months in posterolateral group was 71.9 with the lowest score of 38.4 and the highest score of 96.2. The mean HHS at 6 months in the lateral group was 66.75 with the lowest score of 47.6 and the highest of 85 . Comparison of the HHS at 6 months between the two groups shows that HHS at 6 months is higher in posterolateral approach group with a t value of 1.327 and is statistically nonsignificant with a $\mathrm{p}$ value of 0.19 .

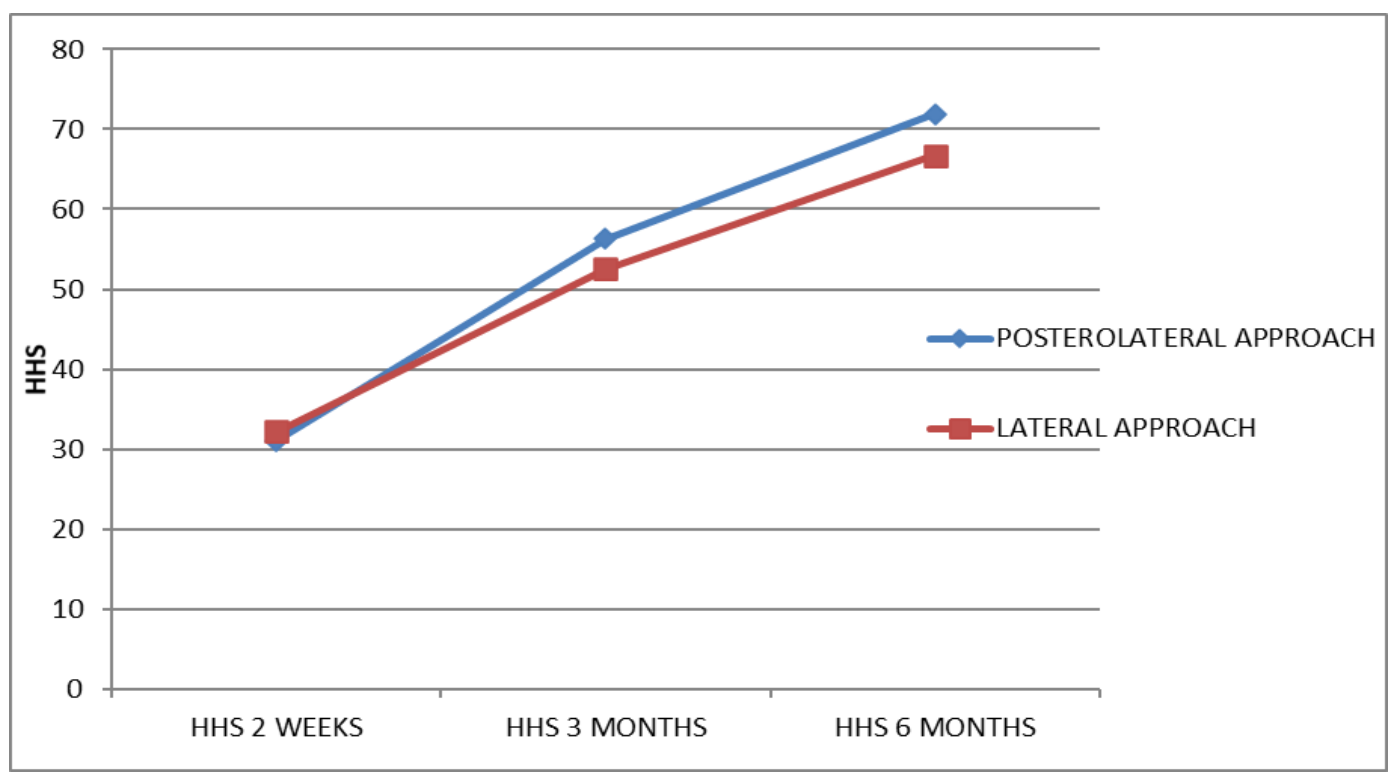

Fig 1: HHS in lateral and posterolateral approach

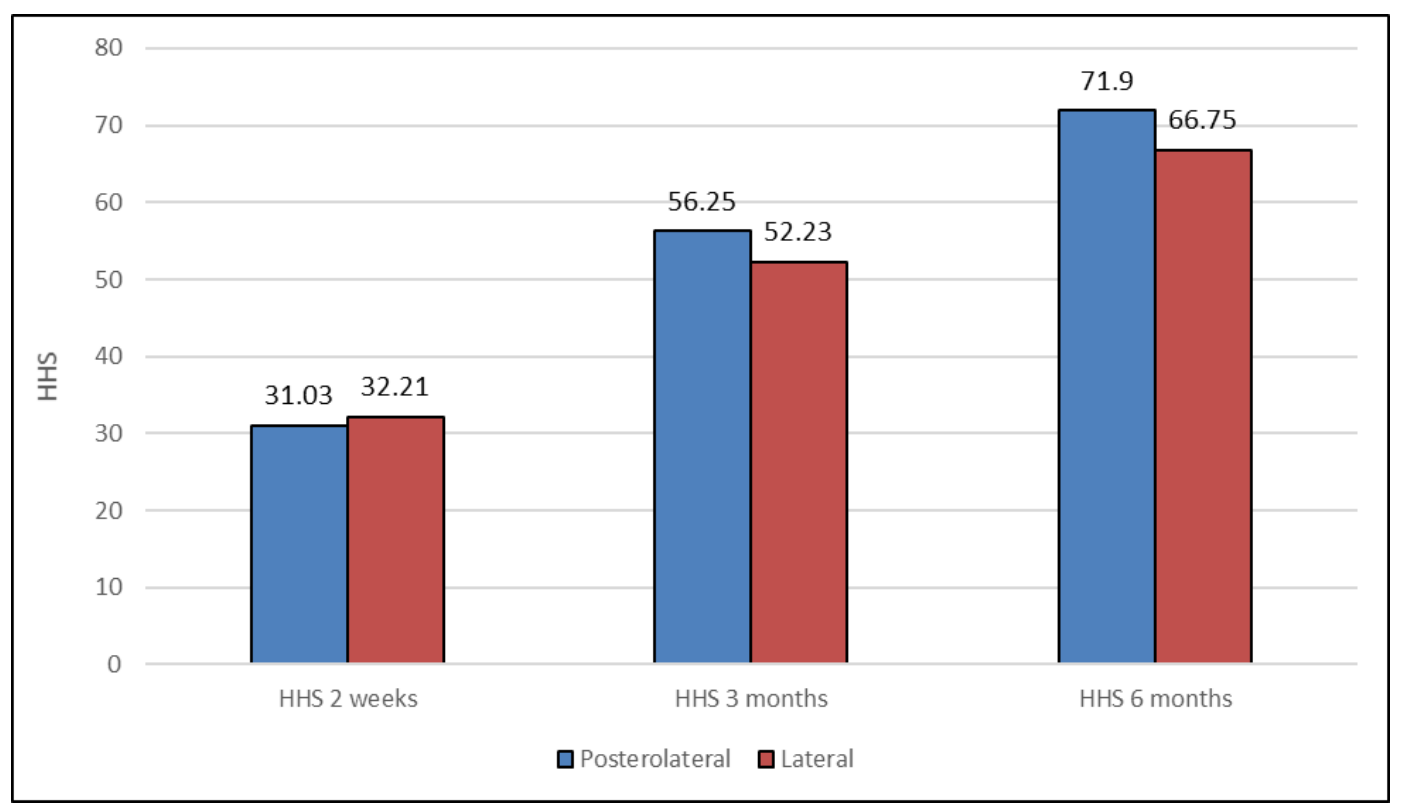

Fig 2: HHS at 2 weeks, 3 months and 6 months $\sim 828 \sim$ 
Comparison of mean value of HHS at 2 weeks, 3 months and 6 months (within the 2 groups)

Comparison of the HHS at 2 weeks to 3 months difference between the two groups shows that HHS is higher in posterolateral approach group with a t value of 2.995 and is statistically significant with a $\mathrm{p}$ value of 0.004 . Comparison of the HHS at 2 weeks to 6 months difference between the two groups shows that HHS is higher in posterolateral approach group with a t value of 2.797 and is statistically significant with a $p$ value of 0.008 . On Comparison of the HHS at 3 months and 6 months, HHS between the two groups is higher in posterolateral approach group with a t value of 1.023 and is statistically non-significant with a $\mathrm{p}$ value of 0.31 .

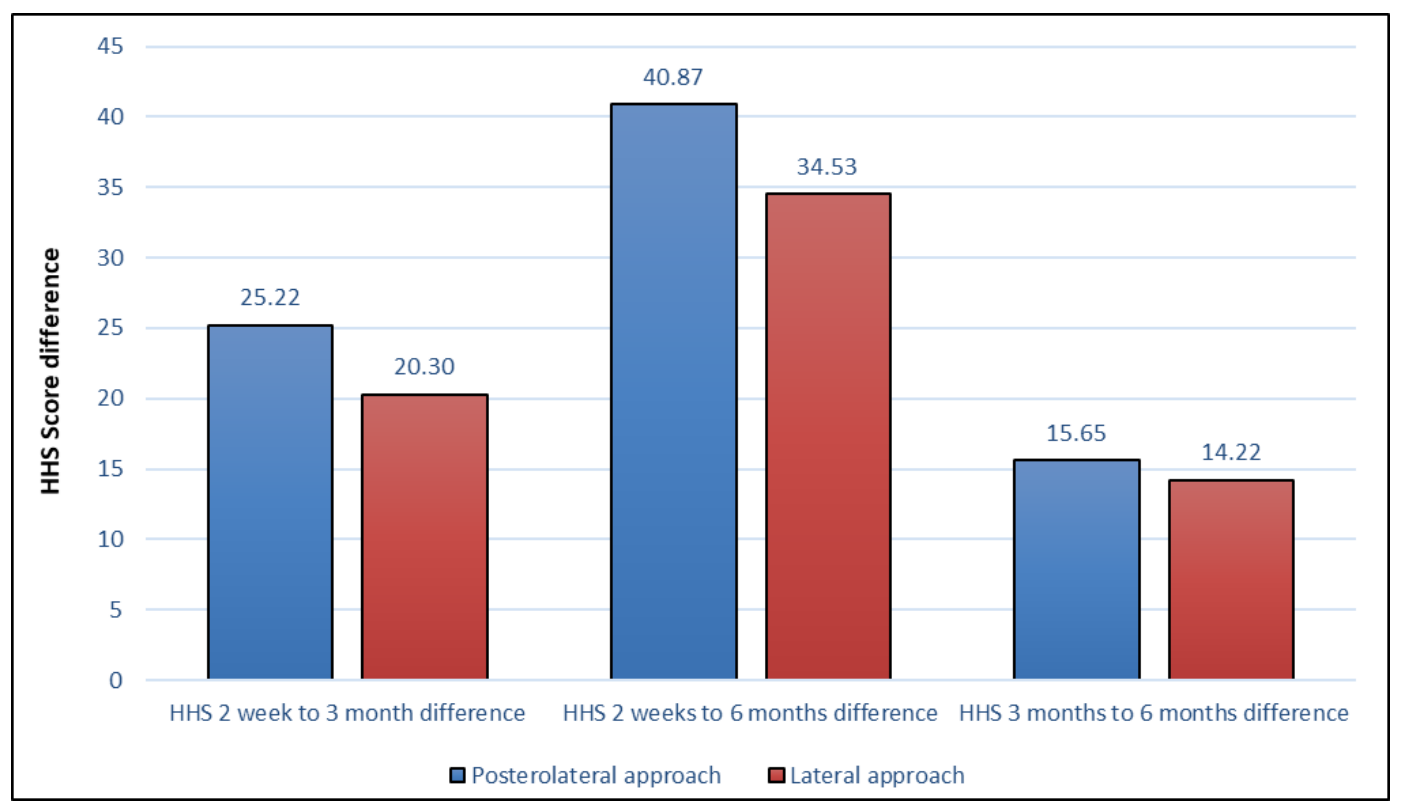

Fig 3: Comparison of HHS at 2 weeks, 3 months and 6 months

\section{Discussion}

Our study included 60 elderly patients diagnosed with nonpathological fracture of femoral neck, operated via posterolateral or lateral approach. We collected clinical profile of patients and followed them up until 6 months, with regular assessment of functional outcome at 2 weeks, 3 months and 6 months.

The highest number of patients was in the age group of 65-74 years. In our study with regards to gender there were equal number of males and females in the posterolateral group. Hip fractures are more common among females, irrespective of age due to the prevalence of osteoporosis. In a 16 year longitudinal study by Elena Lobo et al. ${ }^{[11]}$ in southern Europe, the incidence rate of hip fracture was found to be three times higher in women than in men ${ }^{[12]}$.

The mean duration of surgery was comparable with respect to cemented and uncemented in both study groups, with 73.85 minutes and 73.92 minutes in cemented group in posterolateral and lateral approach groups respectively. In the uncemenetd group, the mean duration of surgery was 64.65 and 65.80 minutes in the posterolateral and the lateral approach groups respectively. However, the duration of surgery was higher in cemented HA in both the study groups as compared to uncemented and was statistically significant.

In a meta-analysis performed by Lin FF et al., shorter operation time was achieved in uncemented HA than that in cemented HA ${ }^{[13]}$, which was same as our study. Santini S, conducted a prospective randomized study to evaluate the clinical and functional outcomes at 12 months of follow up in two groups of patients affected by femoral neck fractures and treated with cemented or cementless bipolar HA. There was no significant difference between the 2 groups of patients regarding most variables, except for duration of operation $(75.00 \pm 22.43$ minutes in cemented group and 56.98 \pm 55.00 minutes in cementless group, $\mathrm{p}<0.001)^{[15]}$.
The mean intraoperative blood loss was calculated as by number of mops used and blood collected in suction minus the total volume of saline used. In posterolateral group was $254.67 \mathrm{ml}$ and that in the lateral approach group was 263.00 $\mathrm{ml}$ and was statistically insignificant.Very few studies have mentioned intraoperative blood loss ${ }^{[16,17]}$.

There was no post-operative infection and posterior dislocation found in any of the patients in either approach groups in our study. Biber et al. conducted a retrospective study on 704 patients and concluded that there was no difference between the posterior approach and the direct lateral approach regarding early surgical complications ${ }^{[18]}$.

In both the approaches, the comparison of mean values of HHS at 2 weeks and 3 months, 2 weeks and 6 months, 3 months and 6 months, a higher HHS value was observed at 3 months and 6 months respectively and these were statistically significant with $\mathrm{p}$ value of $<0.001$. It is also observed that HHS tends to improve with duration of follow-up in the study. At 2 weeks, the score was comparable with 31.03 and 32.21 in posterolateral and lateral groups, which improved to 71.90 and 66.75 at 6 months in posterolateral and lateral groups respectively. In our study at 6 months, satisfactory (excellent + good) results were found in $40 \%$ of posterolateral group while it was $16.66 \%$ in the lateral group.

The final outcome at 6 months follow up in the posterolateral group was excellent (i.e. HHS >90) in 5 patients $(16.66 \%)$, good (i.e.HHS 80-89) in 7 patients $(23.33 \%$ ), fair (i.e. HHS $70-79)$ in 8 patients $(26.66 \%)$ and poor(i.e. HHS <70) in 11 patients $(36.66 \%)$, whereas in lateral group excellent results were not found in any of the patient, good in 5 patients $(16.66 \%)$, fair in 10 patients $(33.33 \%)$ and poor in 13 patients $(43.33 \%)$. Thus, it was observed that final functional outcome (i.e. 6 months) was better in the posterolateral group as compared to the lateral group. In comparison of the difference of HHS between 2 weeks to 3 months, the difference is higher 
in the posterolateral group and is statistically significant. Similarly, the comparison of difference of HHS from 2 weeks to 6 months shows that the difference is higher in the posterolateral group and is statistically significant. In our extensive literature research we did not come across any study comparing these outcomes. Due to limited studies, the importance or clinical value of this statistical significance in our study could not be established.

An Indian study performed by Aparajit et al. ${ }^{[19]}$, in 80 patients of either sex and age between 50-80 years found the mean age of patients was $64.30 \pm 5.39$ years in posterior group and $65.85 \pm 5.64$ years in lateral approach group, with a higher female to male ratio whereas in our study mean age in posterolateral group was 70.97 and 73.03 in lateral group with higher female to male ratio. The average HHS was $85.62 \%$ in posterior group and $83.40 \%$ in lateral group as compared to our study where it was $71.9 \%$ in posterolateral and $66.75 \%$ in lateral group. The difference in HHS may be due to higher mean age and poor functional status of the patients prior to the injury in our study.

\section{Conclusion}

In our study it is concluded that the approach used for HA depends on the preference and training of the surgeon, and results of both the approaches are comparable. There is no significant difference between the lateral and posterolateral approaches in the outcomes of duration of surgery, amount of blood loss, early surgical complications, post-operative prosthesis alignment and the functional outcome. Our study establishes the fact that $\mathrm{HA}$ as an intervention for fracture neck of femur yields satisfactory results and is the treatment of choice. Harris Hip score does not take age and pre-injury functional status of the patient into account. The poor functional outcome in our study was related to increased age and may be related with poor pre injury functional status. The limitations of present study are small sample size and short duration of follow up. There is a better scope for improvement in results with a longer duration of follow up. A larger randomized trial or may be a multicentre trial can improve the interpretation of the results further. We suggest a modified HHS which takes the functional status of the patient prior to the injury into account and comparison of the patients with similar age with a larger sample size to establish HA as a treatment of choice for fracture neck of femur.

\section{Reference}

1. Sozen T, Ozisik L, Basaran NC. An overview and management of osteoporosis. Eur $\mathrm{J}$ Rheumatol 2017;4(1):46-56.

2. Ingle GK, Nath A. Geriatric health in India: Concerns and solutions. Indian J Community Med 2008;33:214-8.

3. Rogmark C, Johnell O. Primary arthroplasty is better than internal fixation of displaced femoral neck fractures: a meta-analysis of 14 randomized studies with 2, 289 patients. Acta Orthop 2006;77(3):359-67.

4. Murphy DK, Randell T, Brennan KL, et al. Treatment and displacement affect the reoperation rate for femoral neck fracture. Clin Orthop Relat Res 2013;471(8):2691702.

5. Yanjiang $\mathrm{Y}$, Juan $\mathrm{W}$, Jiayuan $\mathrm{S}$ et al. Comparison of hemiarthroplasty versus internal fixation in treatment of displaced femoral neck fracture: a meta-analysis. Int $\mathbf{J}$ Clin Exp Med 2016;9(8):15017-25.

6. Hardinge K. The direct lateral approach to the hip. J Bone Jt Surg Br. 1982;64(1):17-9.
7. Moore AT. The self locking metal hip prosthesis. J Bone Jt Surg Br 1957;39-A:811-27.

8. Bush JB. Wilson MO. Dislcoation after hip hemiarthroplasty. Orthopedics. 30:138-44.

9. Jolles BM, Bogoch ER. Posterior versus lateral surgical approach for total hip arthroplasty in adults with osteoarthritis. Cochrane Database Syst Rev. 2006, 3.

10. Parker MJ, Pervez H. Surgical approaches for inserting hemiarthroplasty of the hip. Cochrane Database Syst Rev. 2002, 3.

11. Lobo E, Marcos G, Santabárbara J et al; ZARADEMP Workgroup. Gender differences in the incidence of and risk factors for hip fracture: A 16-year longitudinal study in a southern European population. Maturitas 2017;97:38-43.

12. Cummings SR, Nevitt MC, Browner WS et al. Risk Factors for Hip Fracture in White Women. N Engl J Med. 1995;332:767-73.

13. Lin FF, Chen YF, Chen B et al. Cemented versus uncemented hemiarthroplasty for displaced femoral neck fractures: A meta-analysis of randomized controlled trails. Med Baltim. 2019;(14)98.

14. Ali AE, Aleisa AA, Alsubaie HI et al. Blood Loss Estimation Using Gauze Visual Analogue. Trauma Mon. 2016;21(2):e34131

15. Santini S, Rebeccato A, Bolgan I. Hip fractures in elderly patients treated with bipolar hemiarthroplasty: comparison between cemented and cementless implants. J Orthopaed Traumatol 2005;6:80.

16. Guo WJ, Wang JQ, Zhang WJ et al. Hidden blood loss and its risk factors after hip hemiarthroplasty for displaced femoral neck fractures: A cross-sectional study. Clin Interv Aging. 2018;13:1639-45.

17. Sehat KR, Evans R, Newman JH. How much blood is really lost in total knee arthroplasty? Correct blood loss management should take hidden loss into account. Knee. 2000;7(3):151-5.

18. Biber R, Brem M, Singler K et al. Dorsal versus transgluteal approach for hip hemiarthroplasty: An analysis of early complications in seven hundred and four consecutive cases. International Orthopaedics. 2012;36(11): 2219-23.

19. Aparajit P, Yadav V, Koichade M. A Comparative Study of Posterior Approach versus Lateral Approach in Surgical Management of Intra-capsular Neck Femur Fractures. Int J Biomed Adv Res 2016;8(3):110-225. 